\title{
Reusable holographic velocimetry system based on polarization multiplexing in Bacteriorhodopsin
}

\author{
W. D. Koek ${ }^{\mathrm{a}}$, V. S. S. Chan ${ }^{\mathrm{b}}$, T. A. Ooms ${ }^{\mathrm{b}}$, N. Bhattacharya ${ }^{\mathrm{a}}$, J. Westerweel ${ }^{\mathrm{b}}$, and J. J. M. Braat ${ }^{\mathrm{a}}$ \\ ${ }^{a}$ Optics Research Group, Delft University of Technology, Lorentzweg 1, 2628 CJ, Delft, \\ The Netherlands. \\ ${ }^{\mathrm{b}}$ Laboratory for Aero- and Hydrodynamics, Delft University of Technology, Leeghwaterstraat 21, \\ 2628 CA, Delft, The Netherlands.
}

\begin{abstract}
We present a novel holographic particle image velocimetry (HPIV) system using a reversible holographic material as the recording medium. In HPIV the three-dimensional flow field throughout a volume is detected by adding small tracer particles to a normally transparent medium. By recording the particle distribution twice with a known time shift the displacement and the velocity of the tracer particles can be retrieved. From this information the instantaneous threedimensional flow field can be found.

Our measurement system records double exposure particle holograms in a film based on the photo-chromic protein bacteriorhodopsin (BR). Polarization multiplexing is used to separate the two constituent holograms. We believe it is the first time that this type of multiplexing is used in (particle) velocimetry measurements. By using a polarization sensitive material we are able to simplify our setup and increase the storage capacity of our holographic medium.

$\mathrm{BR}$ is a fully reversible recording material that does not require any chemical processing. This allows for fast experiments that require minimal operator involvement. A full measurement cycle can typically be completed within several minutes.

We present our experimental system in detail and we will discuss how the material and optical properties of BR affect the holographic recording system. We will point out the advantages, disadvantages, and practical issues involved when working with BR.
\end{abstract}

Keywords: holography, bacteriorhodopsin, br, polarization holography, polarization multiplexing, holographic particle image velocimetry, HPIV

\section{INTRODUCTION}

Particle image velocimetry (PIV) is a commonly used technique for the study of fluid flows. ${ }^{1}$ In its basic form PIV yields planar two-component flow field information. Although the PIV technique is continuously improved and has been extended to perform three-dimensional measurements, ${ }^{2,3}$ it has thus far been impossible to perform truly instantaneous three-dimensional three-component flow measurements. The need for such measurements suggests the use of holographic imaging systems, and much effort is currently focussed toward the development of such systems. Over the past decade several successful holographic particle image velocimetry (HPIV) systems have been presented. $^{4-8}$ One major drawback of these systems is that they rely on silver-halide film as the holographic recording medium. The chemical processing that is required with such films causes shrinkage, leading to distortion of the reconstructed particle images. Also the chemical processing requires a skilled person, and is time consuming. As a result the availability of HPIV has not yet revolutionized the way in which flow measurements are performed. Obviously any commercially available HPIV system must be easily usable outside the specialized laboratory it was developed in. Alternative to filmbased HPIV much effort is currently focussed towards the development of digital holographic flow measurement systems. Although the technique has many practical advantages its major disadvantage is the limited space-bandwidth of current CCD sensors. Whereas a film-based system may record the position data of $10^{6}$ particles, it can be estimated that a CCD sensor with approximately $10^{8}$ pixels would be required to accurately reconstruct such a particle field. ${ }^{9}$ Such sensors are not yet available, thereby limiting the possibilities of digital HPIV. Considering the above, Barnhart et al 
realized that a practical HPIV system must use a holographic material that does not require chemical development, and they suggested the use of bacteriorhodopsin (BR). ${ }^{10}$ In this paper we will discuss the design of the first operational HPIV system to use BR as the recording material. The presented system is also the first of its kind to use polarization multiplexing to separate the two constituent images. Prior to discussing our particular system we will first give an overview of the design criteria relating to HPIV systems.

\section{HPIV DESIGN CRITERIA}

The goal of any HPIV system is to perform three-dimensional flow measurements. This is accomplished by adding small tracer particles to a normally transparent fluid or gas. Under the assumption that the particles accurately follow the flow of the surrounding medium, the flow in the medium can be analyzed by following the particles as they move throughout the measurement volume. Once the position of the particles at two different instances has been recorded the flow field can be deduced. With low seeding densities the approach would be to identify the movement of each individual particle. This technique is referred to as particle tracking velocimetry (PTV). Alternatively, as the seeding density gets higher it is possible to retrieve the average motion of a group of particles by correlating a certain volume at the time of the first recording with the same volume at the time of the second recording. This technique is commonly referred to as particle image velocimetry (PIV). Whereas in two-dimensional imaging techniques there is a clear distinction between PTV and PIV, in the holographic community people tend to talk about HPIV even when the analysis is based on particle tracking. A measurement thus consists of at least two recordings. Based on the type of flow that is analyzed the time between the two recordings typically ranges from a few microseconds to several tens of milliseconds. When using a double-exposure digital camera, as is the case in regular PIV or digital HPIV, the two recordings may be easily performed using a double laser pulse that travels the same beam path each time. In film based HPIV the two recordings must be multiplexed. After all, they must be able to be reconstructed individually. Most HPIV systems employ angular multiplexing. A disadvantage of this type of multiplexing is that two additional beam paths are required, one for the recording and one for the reconstruction. This makes the system more bulky, more sensitive to misalignment, and therefore, in our opinion, less suitable for integration and application in a possible future commercial system.

Obviously the holograms need to be recorded with a pulsed laser with a good coherence length. In our laboratory we use a seeded Spectra Physics double-cavity frequency doubled Nd:YAG laser (532 nm, $7 \mathrm{~ns}, 300 \mathrm{~mJ}$ pulse). The wavelength of $532 \mathrm{~nm}$ is commonly used for HPIV, as it is readily available. Any candidate material for HPIV must therefor be sensitive at this wavelength. The laser is by far the single most expensive part of the holographic system, and it thus pays to have a more sensitive material as this allows the use of a lower power, and therefor less expensive laser. Typically it can be said that the maximum available laser power ranges between $300 \mathrm{~mJ}$ and $1 \mathrm{~J}$ per pulse. With a typical hologram aperture size of $\sim 50 \mathrm{~cm}^{2}$, the typical sensitivity of any candidate material must be better than $10 \mathrm{~mJ} / \mathrm{cm}^{2}$.

As mentioned earlier the chemical development associated with silver-halide film adversely affects the quality of the reconstructed particle images, and is far from practical for implementation in any user-friendly system. Ideally a HPIV system should have a reusable holographic film that does not require any development.

The basic requirements for a candidate material are summarized in Table 1. In the next chapter we will first give a brief introduction to BR, after which its properties (already displayed in Table 1) will be discussed in relation to the given design criteria.

Table 1. Requirements of holographic material for HPIV

\begin{tabular}{ccccc}
\hline \hline Preferred properties & Silver Halide & $\begin{array}{c}\text { Dichromated } \\
\text { Gelatin }\end{array}$ & $\begin{array}{c}\text { Photo-polymer } \\
\text { (Aprilis) }\end{array}$ & BR \\
\hline \hline No development required & - & - & + & + \\
\hline Re-usable & - & - & - & + \\
\hline Sensitive to 532 $\mathrm{nm}$ & + & + & + & + \\
\hline Required exposure $<10 \mathrm{~mJ} / \mathrm{cm}^{2}$ & + & - & + & + \\
\hline Alternative multiplexing scheme & - & - & + \\
\hline
\end{tabular}




\section{BACTERIORHODOPSIN}

BR is a photochromic protein which, since its discovery 3 decades ago, has increasingly been finding its way into a large variety of technical applications. ${ }^{11} \mathrm{BR}$ is found inside halobacteria in the form of a two dimensional crystalline lattice, known as purple membrane. Halobacteria produce BR as the key protein for their photosynthetic capabilities, and by means of evolution BR has acquired a high quantum yield of $64 \%$ for its primary photoreaction. Combined with other interesting photophysical properties such as a large spectral shift of $150 \mathrm{~nm}$ between the two key states (B, M) in the BR photocycle, a very high spatial resolution of several thousand line pairs per millimetre, and $100 \%$ reversibility, BR is highly attractive as a rewritable holographic medium. Apart from wildtype BR several genetic variants with optimized characteristics exist. The variant $\mathrm{D} 96 \mathrm{~N}$ has an increased lifetime of the meta-stable $\mathrm{M}$ state, allowing for a longer holographic reconstruction. The red-shifted variant $\mathrm{D} 85 \mathrm{~N}$, also known as blue membrane, can store information for several days, or even months, but this variant has much lower sensitivity. The photocycle for D96N BR is shown in Figure 1. In this figure the various states of the molecule are indicate with capital letters. Black arrows indicate thermal transitions (all of which happen at the given rate at room temperature). Gray arrows indicate photo-induced transitions (those that happen upon absorption of light). For each of the intermediate states the peak of the absorption band (in nm) is indicated. The absorption band is broad enough such that all of the intermediates, except M, absorb at $532 \mathrm{~nm}$. For most practical applications the complex photocycle of Figure 1 may be simplified by means of the two-state model as shown in Figure 2. Initially the BR molecule is in the ground state B. Upon excitation with light of $532 \mathrm{~nm}$ the molecule will make a transition to the $\mathrm{M}$ state. The typical decay time from $\mathrm{M}$ to $\mathrm{B}$ is 80 seconds, this means that information can only be stored for a limited time. Alternatively, one may intentionally erase all information be exposing the molecule to light of $410 \mathrm{~nm}$, this will trigger a M-to-B transition instantly. The photo-physical properties of the molecule change quite significantly when changing from one state to the other. Whereas the B state is a good absorber for light of 532 $\mathrm{nm}$, the M state practically does not absorb at this wavelength. Also there is a change of refractive index in the order of $10^{-3}$ between both states. These changes in the properties of the material obviously allow for the (temporary) storage of information in the material. In the case of holography a combined phase-amplitude grating may be recorded in BR.

BR has another interesting feature: the molecule is sensitive to the polarization of the illuminating light. The likelihood of a photo-induced transition depends on $\kappa_{1}(\varphi)$, the absorption cross-section for BR at the wavelength of the exciting light. The angular dependence of $\kappa_{1}(\varphi)$, describing the recording anisotropy of the BR molecule, is given by:

$\kappa_{1}(\varphi)=\kappa_{1}\left(\cos ^{2} \varphi+k_{1} \sin ^{2} \varphi\right)$

where $\kappa_{1}$ is the absorption cross-section for the molecule's long axis, $\varphi$ is the angle between the molecule's long axis and the plane of polarization of the exciting light, and the dichroic ratio $k_{1}=0.04 .{ }^{12}$ Recording with polarized light will therefore lead to a photo-induced anisotropic distribution of BR molecules in the B state and the M state.

Now that the basic properties of BR have been described we will discuss how they match the criteria of a HPIV candidate material as given in Table 1 .

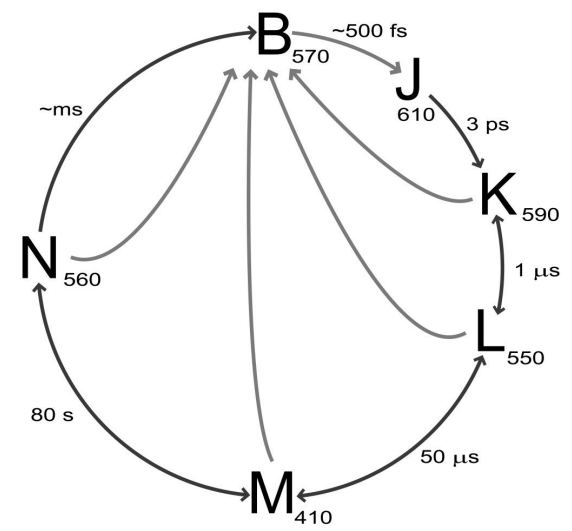

Figure 1: Photocycle for D96N BR. Black arrows indicate a thermal transition. Gray arrows indicate photo-induced transitions. The absorption peak for each photointermediates is shown.

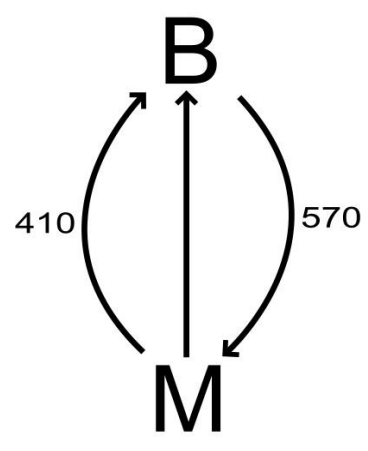

Figure 2: Simplified two-state model of the BR photo-cycle. 


\section{No development necessary}

As discussed earlier the sequence of conformational changes leading the molecule through the states $\mathrm{B} \rightarrow \mathrm{J} \rightarrow \mathrm{K} \rightarrow \mathrm{L} \rightarrow \mathrm{M} \rightarrow \mathrm{N} \rightarrow \mathrm{B}$ is solely triggered by a single photo-induced transition (B $\rightarrow \mathrm{J}$ ). Approximately 50 microseconds after the illumination occurred the molecule will inhabit the $\mathrm{M}$ state. At this point the photo-physical properties of the molecule have changed significantly and the holographic reconstruction may be started. BR thus only requires illumination to record the information and no further chemical or physical treatment. Also, the recording process may be considered instantly for most practical purposes. Because there is no development or fixing involved this also implies that the material remains sensitive. When performing a holographic reconstruction the illumination will continue to trigger $\mathrm{B} \rightarrow \mathrm{M}$ transitions thereby effectively erasing the stored information. Also, the thermal decay of BR will lead to erasure of the stored information. At room temperature a hologram can be typically reconstructed for several minutes. When cooling the film to $-10^{\circ} \mathrm{C}$ the information may be stored up to 1 hour. However, BR should be considered as a temporary storage medium, or holographic buffer.

\section{Re-usable}

The cyclic nature of the sequence of conformational changes upon illumination implies that the molecule returns to its original state, thereby being fully re-usable. In practice a small decrease in the optical density of the film is observed over time. However over at least one million cycles the material has more or less constant holographic performance. ${ }^{13}$ To ensure a maximum lifetime of the film it is recommended to not expose a BR film to light with a wavelength smaller than $400 \mathrm{~nm}$. The BR protein absorbs around $380 \mathrm{~nm}$, and this absorption may cause damage thereby decreasing the performance of the film. As was mentioned earlier, light of a wavelength around $410 \mathrm{~nm}$ may be used to intentionally erase the film $(\mathrm{M} \rightarrow \mathrm{B})$. Typically a recording cycle consists of (1) recording the information with a pulsed laser [ 10 ns], (2) reconstruct the information with a cw laser[ 1 minute], (3) illuminate film with $410 \mathrm{~nm}$ LED [10 seconds], after which the entire process may start again. The material can practically be thus considered to be fully re-usable with a measurement turn-around time in the order of minutes, if necessary seconds.

\section{Sensitive to $532 \mathrm{~nm}$}

As was indicated in Figure 1 the B state has its absorption maximum at $570 \mathrm{~nm}$. However the broadness of the spectrum ensures that at $532 \mathrm{~nm}$ the sensitivity has only reduced to $70 \%$ of its maximum value. ${ }^{14}$ The sensitivity of the $\mathrm{M}$ state at $532 \mathrm{~nm}$ is less than $1 \%$ of the sensitivity of the B state. BR thus has excellent photo-physical properties when recording at $532 \mathrm{~nm}$.

\section{Required exposure $<10 \mathrm{~mJ} / \mathrm{cm}^{2}$}

The sensitivity of a BR film with optical density $\mathrm{OD}_{570}=1.5$ when recording with a pulsed laser was found to be $\sim 5$ $\mathrm{mJ} / \mathrm{cm}^{2}$. Obviously this is the combined fluence of both the reference and the object beam. Due to the low fraction of the object illumination beam that will be scattered, and thereby forms the actual object wave, it is typically not feasible to achieve equal object and reference intensity. With HPIV it will be often the case that the reference beam has much higher intensity at the plane of the hologram than the object beam. In the remainder of this paragraph we will discuss how the power should be distributed between the reference and the object beam to ensure maximum diffraction.

The interference between reference wave and object wave leads to a spatially dependent intensity distribution. If the two beams have equal intensity the intensity distribution will reach a full null when the two beams are out of phase, and is therefor fully modulated. If the two beams do not have equal strength the intensity will be modulated around an offset. Because the diffraction efficiency of a hologram depends on the modulated intensity, it is the holographers aim to make the modulated intensity as large as possible. It is a general misconception that this is always achieved when the object and reference beam intensity are made equal.

In an application where the laser power $\mathrm{P}$ is limited, the available power will have to be divided between the reference beam and the object beam. For the reference beam intensity at the plane of the hologram we may write:

$$
I_{r}=\frac{\rho P}{A_{R}},
$$


where $\rho$ denotes the fraction of $P$ that enters the reference beam, and $A_{r}$ is the area of the reference beam at the holographic film. For the reference beam amplitude follows:

$$
R=\sqrt{\frac{\rho P}{A_{R}}} .
$$

In a similar manner we find for the amplitude of the object beam:

$$
O=\sqrt{\frac{\gamma(1-\rho) P}{A_{o}}},
$$

where $\gamma$ is the scattered fraction of the object beam that reaches the holographic film, and $A_{o}$ is the area of the object beam at the holographic film. For the modulated intensity can then be found:

$$
\begin{aligned}
M & =(O+R)^{2}-(O-R)^{2} \\
& =4 O R \\
& =4 P \sqrt{\frac{\gamma}{A_{r} A_{o}} \sqrt{\rho-\rho^{2}}} .
\end{aligned}
$$

In a given application $P$ is limited by the available laser power, $A_{r}$ and $A_{o}$ are determined by the setup, and $\gamma$ is fixed for a given object. The modulated intensity will thus reach a maximum when $\left(\rho-\rho^{2}\right)^{1 / 2}$ is maximal. This occurs when $\rho=0.5$. Thus the modulated intensity reaches a maximum when the object beam and the reference beam contain equal power at the point where they are separated. This does not necessarily result in an equal intensity at the hologram plane. When expanding the object beam, or when working with a very weak scattering object, no adjustment of the reference-toobject ratio is required, as is often falsely assumed.

Apart from modulated intensity, the total exposure level during the recording also affects the resulting diffraction efficiency. If, due to choosing $\rho=0.5$ the required exposure level of $5 \mathrm{~mJ} / \mathrm{cm}^{2}$ is not met, the film may be pre- or postbleached. If due to choosing $\rho=0.5$ the required exposure level of $5 \mathrm{~mJ} / \mathrm{cm}^{2}$ is exceeded then, despite of the resulting loss of modulation, more light should be directed towards the object beam until the exposure requirements are met.

\section{Alternative multiplexing scheme}

The polarization sensitivity of the BR molecule allows for the recording of polarization holograms. This allows for the recording of a hologram even when the object and reference beam have orthogonal polarizations. In Table 2 and Table 3 the polarization state of the -1 diffracted order (constructing a real image), and the normalized intensity of the -1 diffracted order are shown for various combinations of reference, object, and reconstruction polarization. ${ }^{15}$ The intensity has been normalized with respect to the case when all polarizations are vertical. In Table 3 can be seen that if the hologram is recorded using left hand circularly polarized (LHCP) light for the reference beam and right hand circularly polarized (RHCP) light for the object beam, no image is reconstructed when the reconstruction polarization is RHCP, yet that a high intensity image is reconstructed when the reconstruction polarization is LHCP. Alternatively, when the hologram is recorded using RHCP for the reference beam and LHCP for the object beam, no image is reconstructed when the reconstruction polarization is LHCP, yet that a high intensity image is reconstructed when the reconstruction polarization is RHCP. This obviously allows for an alternative form of multiplexing. After all, by switching the reconstruction polarization between RHCP and LHCP either the first or second recorded image may be reconstructed. 
Table 2. Polarization and normalized intensity of the diffracted -1 order when recording and reconstructing with different combinations of linearly polarized light. ${ }^{15}$

\begin{tabular}{|c|c|c|c|c|}
\hline $\mathrm{R}$ & $\mathrm{O}$ & Recon. & $\mathrm{P}_{-1}$ & $\mathrm{I}_{-1}$ \\
\hline \multirow{4}{*}{$\downarrow$} & \multirow{4}{*}{ } & $\downarrow$ & $\uparrow$ & 1.0 \\
\hline & & $\leftrightarrow$ & $\leftrightarrow$ & 0.27 \\
\hline & & (R) & $\left.R^{*}\right)$ & 0.73 \\
\hline & & (L) & $(\mathbb{t}$ & 0.68 \\
\hline \multirow{4}{*}{$\longleftrightarrow$} & \multirow{4}{*}{$\longleftrightarrow$} & $\downarrow$ & $\uparrow$ & 0.25 \\
\hline & & $\longleftrightarrow$ & $\hookrightarrow$ & 0.86 \\
\hline & & (R) & $\left.R^{*}\right)$ & 0.52 \\
\hline & & (L) & 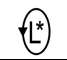 & 0.56 \\
\hline \multirow{4}{*}{$\downarrow$} & \multirow{4}{*}{$\longleftrightarrow$} & $\downarrow$ & $\hookrightarrow$ & 0.27 \\
\hline & & $\longleftrightarrow$ & $\downarrow$ & 0.31 \\
\hline & & R) & (D) & 0.29 \\
\hline & & (L) & (R) & 0.25 \\
\hline \multirow{4}{*}{$\longleftrightarrow$} & \multirow{4}{*}{ I } & $\downarrow$ & $\hookrightarrow$ & 0.25 \\
\hline & & $\longleftrightarrow$ & $\uparrow$ & 0.25 \\
\hline & & (R) & (L) & 0.26 \\
\hline & & (D) & R & 0.25 \\
\hline
\end{tabular}

Table 3. Polarization and normalized intensity of the diffracted -1 order when recording and reconstructing with different combinations of circularly polarized light. ${ }^{15}$

\begin{tabular}{|c|c|c|c|c|}
\hline $\mathrm{R}$ & $\mathrm{O}$ & Recon & $\mathrm{P}_{-1}$ & $\mathrm{I}_{-1}$ \\
\hline \multirow{4}{*}{ (R) } & \multirow{4}{*}{ R } & $\downarrow$ & $\uparrow$ & 0.90 \\
\hline & & $\longleftrightarrow$ & $\longleftrightarrow$ & 0.89 \\
\hline & & R) & (R) & 0.88 \\
\hline & & (D) & (D) & 0.87 \\
\hline \multirow{4}{*}{ (L) } & \multirow{4}{*}{ (L) } & $\downarrow$ & $\uparrow$ & 0.87 \\
\hline & & $\longleftrightarrow$ & $\leftrightarrow$ & 0.82 \\
\hline & & (R) & (R) & 0.88 \\
\hline & & (D) & (D) & 0.92 \\
\hline \multirow{4}{*}{ (L) } & \multirow{4}{*}{ R } & 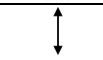 & (R) & 0.49 \\
\hline & & $\longleftrightarrow$ & (R) & 0.59 \\
\hline & & (R) & $x$ & 0 \\
\hline & & (L) & (R) & 1.00 \\
\hline \multirow{4}{*}{ (R) } & \multirow{4}{*}{ (L) } & & (D) & 0.47 \\
\hline & & $\longleftrightarrow$ & (L) & 0.46 \\
\hline & & (R) & (D) & 0.90 \\
\hline & & (D) & $x$ & 0 \\
\hline
\end{tabular}




\section{HPIV USING BR}

In the previous chapter it has been shown that BR is a suitable material for use in a HPIV system. Its sensitivity (both wavelength and power) allow the material to be used with readily available laser systems. Its self-development, reusability, and potential of polarization multiplexing give BR an advantage over silver-halide materials when it comes to integration in a future commercial HPIV system. To demonstrate the capabilities of BR for HPIV we have developed a fully operational system. The system is schematically shown in Figure 3.

When recording a particle hologram light from a pulsed Nd:YAG laser is split into an object and a reference wavefront using a half wave plate and a Glan laser polarizer. The polarization of the object beam may be adjusted using Pockels cell P1, after which the object illumination beam falls onto a tank filled with water containing tracer particles. Light scatters from the particles and falls onto the BR film. The reference polarization may be adjusted using Pockels cell P2. The reference beam is then expanded and collimated using lenses L1 and L2 after which it falls onto the BR film. The interference pattern between $\mathrm{O}$ and $\mathrm{R}$ will be recorded in the BR as a modulation of the molecules in the B state and the $\mathrm{M}$ state. Two recordings of the particle field are made. For the first the Pockels cells are adjusted such that R has LHCP at the plane of the hologram, and $\mathrm{O}$ has RHCP. For the second recording the Pockels cell are adjusted such that R has RHCP at the plane of the hologram, and $\mathrm{O}$ has LHCP. After the recording of the two multiplexed holograms has been performed the BR film is transferred to the reconstruction set-up.

A cw Nd:YAG is used for the reconstruction. Using a quarter wave plate and a moveable half wave plate the polarization of the reconstruction beam may switch between LHCP and RHCP. Using a spatial filter and lens L3 the reconstruction beam is expanded and collimated. A CCD is used to scan the volume where the real image of the particle field is reconstructed. If the reconstruction beam has LHCP then the first recorded particle field is reconstructed (see Figure 4). If the reconstruction beam has RHCP then the second recorded particle field is reconstructed. By scanning the $\mathrm{CCD}$ and alternating the polarization of the reconstruction beam, the reconstruction procedure thus results in 2 threedimensional intensity maps, each belonging to a different instance of the particle field. From these two intensity maps the three-dimensional flow may be deduced. Such an analysis will not be discussed further at this moment. The threedimensionality of the measurement technique is illustrated in Figure 5 and Figure 6.

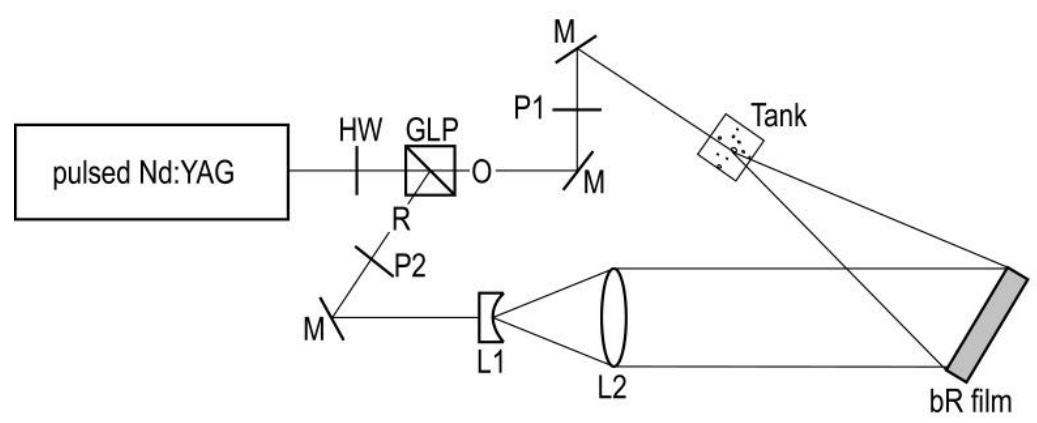

(Recording geometry)

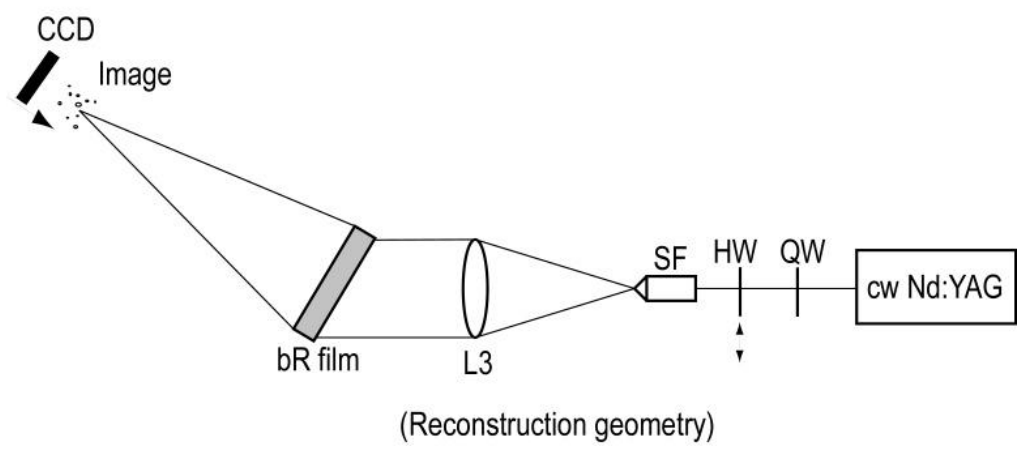

Figure 3. Schematic of HPIV system based on BR. HW: half wave plate; GLP: Glan laser polarizer; O: object wavefront; R: reference wavefront; M: mirror; Px: Pockels cell; Lx: lens; QW: quarter wave plate; SF spatial filter. 


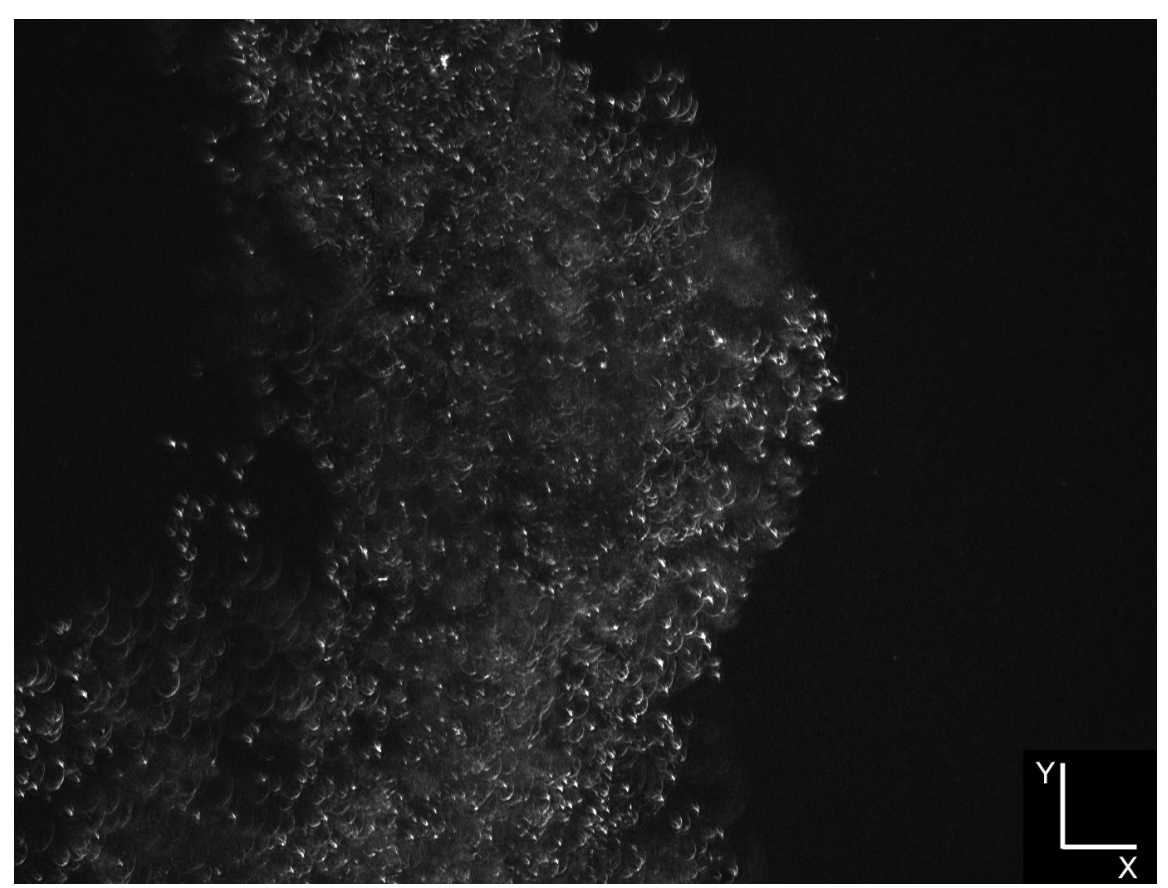

Figure 4. Intensity of the reconstructed particle field in a single plane within a particle seeded jet.

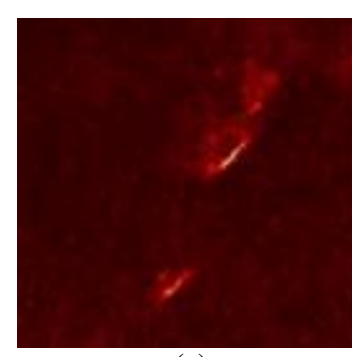

(a)

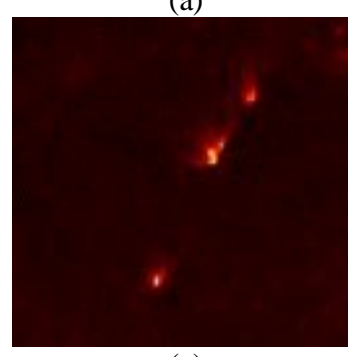

(e)

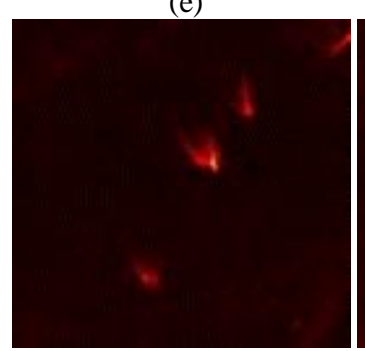

(i)

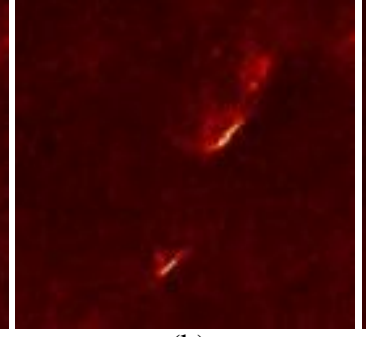

(b)

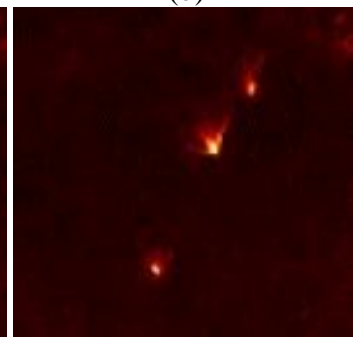

(f)

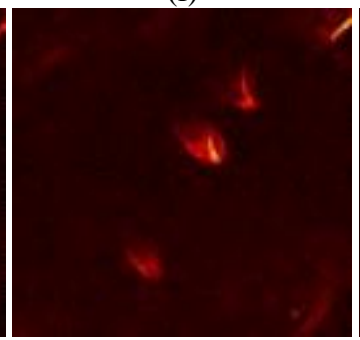

(j)

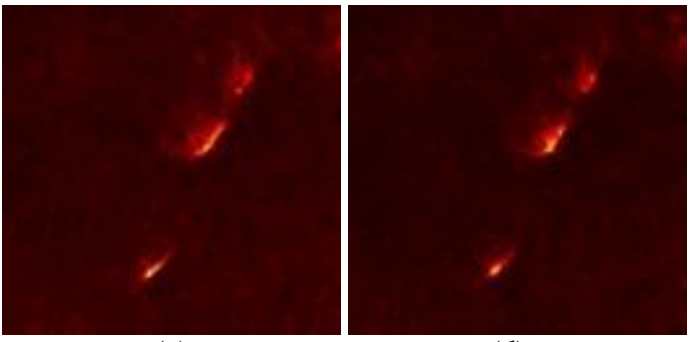

(c)

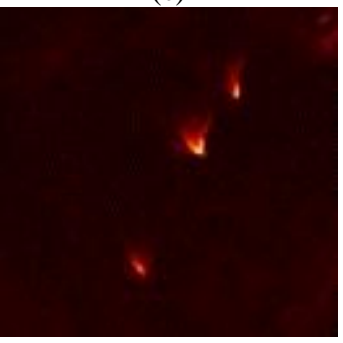

(g)

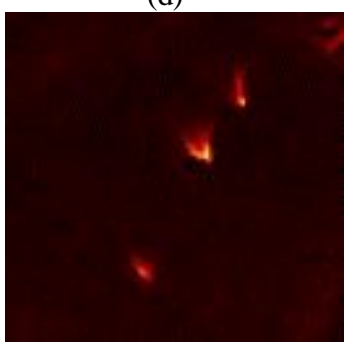

(h)

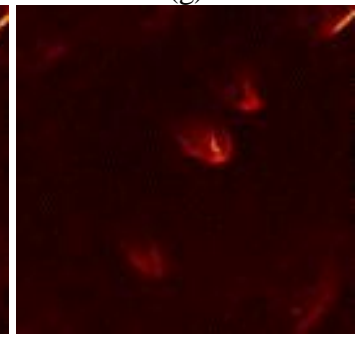

(k)

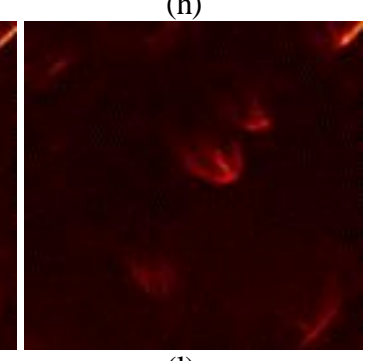

(l)

Figure 5. Focussing of three particles obtained by scanning the CCD. Between each subsequent image the CCD was shifted $50 \mu \mathrm{m}$ in the z-direction. 


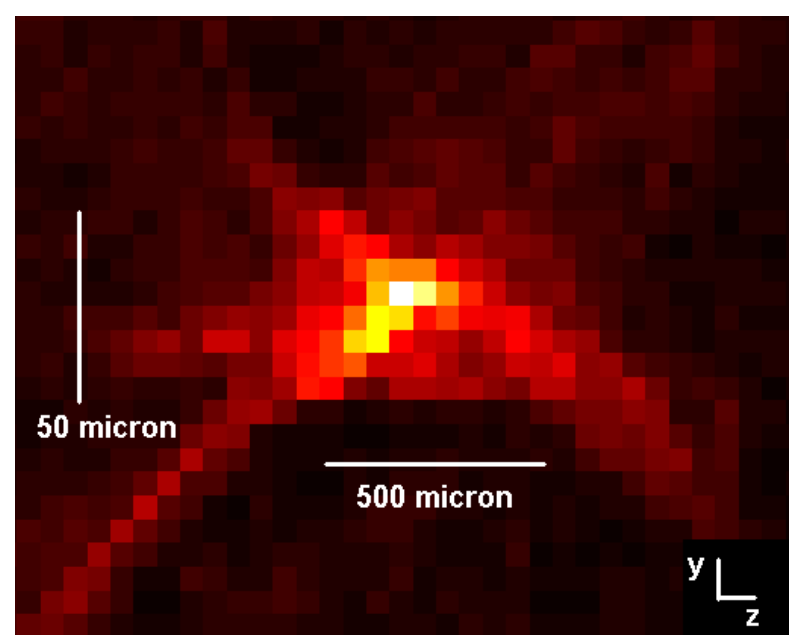

Figure 6. Focussing of a single particle obtained by scanning the CCD. The depth of focus is approximately $50 \mu \mathrm{m}$.

\section{CONCLUSION}

We have discussed how the usage of silver-halide materials in HPIV systems has limited the practical application of such systems for performing flow measurements. A series of criteria were presented based on which it is believed that a holographic velocimetry system is more likely to find its way outside the specific laboratory it has been developed in. These criteria were (a) no development necessary, (b) re-usable, (c) sensitive to $532 \mathrm{~nm}$, (d) required exposure $<10 \mathrm{~mJ} / \mathrm{cm}^{2}$, and (e) possibly support alternative form of multiplexing. The properties of BR were discussed, and the performance of BR was compared to the desired specifications. BR turns out to be an excellent candidate material for use in future HPIV systems. We have presented a HPIV system based on BR and have shown a reconstructed particle field. From the two sets of three-dimensional intensity distributions that were acquired with the system it is possible to obtain the three-dimensional flow field.

\section{ACKNOWLEDGMENTS}

This work has been funded by the Foundation for Fundamental Research on Matter (FOM), the Netherlands. The authors thank Peter Somers for the generous loan of equipment. Furthermore we thank Prof. Norbert Hampp (PhilippsUniversität Marburg) and Donald Barnhart for their support on BR related issues.

\section{REFERENCES}

1. R.J. Adrian, "Particle-imaging techniques for experimental fluid mechanics", Ann. Rev. Fluid Mech. 23, 261-304, 1991.

2. C Brücker, "Digital-particle-image-velocimetry (DPIV) in a scanning light-sheet: 3-D starting flow around a short cylinder", Exp. Fluids 19, 255-263, 1995.

3. T. Hori and J. Sakakibara, "High-speed scanning stereoscopic PIV for 3D vorticity measurement in liquids", Meas. Sci. Technol. 15, 1067-1078, 2004.

4. D. H. Barnhart, R. J. Adrian, and G. C. Papen, "Phase conjugate holographic system for high resolution particle image velocimetry", Appl. Opt. 33, 7159-7170, 1994.

5. J. Zhang, B. Tao, and J. Katz, "Turbulent flow measurement in a square duct with hybrid holographic PIV", Exp. Fluids 23, 373 - 381, 1997.

6. Y. Pu and H. Meng, "An advanced off-axis holographic particle image velocimetry (HPIV) system", Exp. Fluids 29, $184-197,2000$.

7. S. Herrmann, H. Hinrichs, K. D. Hinsch , and C. Surmann, "Coherence concepts in holographic particle image velocimetry", Exp. Fluids 29, S108-S116, 2000. 
8. K.D. Hinsch, "Holographic particle image velocimetry - Review Article", Meas. Sci. Technol. 13, R61-R72, 2002.

9. H. Meng, G. Pan, Y. Pu, and S.H. Woodward, "Holographic particle image velocimetry: from film to digital recording", Meas. Sci. Technol. 15, 673-685, 2004.

10. D. H. Barnhart, N. Hampp N. A. Halliwell, and J. M. Coupland, "Digital holographic velocimetry with bacteriorhodopsin (BR) for real-time recording and numeric reconstruction," presented at the $11^{\text {th }}$ International Symposium on Applications of Laser Techniques to Fluid Mechanics, Lisbon, Portugal, July 2002.

11. N. Hampp, "Bacteriorhodopsin as a photochromic retinal protein for optical memories", Chem. Rev. 100, 17551776, 2000.

12. N.M. Burykin, E. Ya. Korchemskaya, M.S. Soskin, and V.B. Taranenko, "Photoinduced anisotropy in bio-chrom films", Opt. Comm. 54, 68-70, 1985.

13. Manufacturer's specifications. Munich Innovative Biomaterials (MIB) GmbH, Hans-Meerwein-Strasse, Gebaude J, D-35032 Marburg, Germany. www.mib-biotech.de

14. C. Gergely, L. Zimányi, and G. Váró, "Bacteriorhodopsin intermediate spectra determined over a wide pH range", J. Phys. Chem. B 101, 9390-9395, 1997.

15. W.D. Koek, V.S.S. Chan, N. Bhattacharya, J.J.M. Braat, and J. Westerweel, "Polarization Multiplexing Based on Photo-Induced Anisotropies in Bacteriorhodopsin", Proceedings of the International Workshop on Holographic Metrology in Fluid Mechanics, J. Coupland, ed., 123-129, Loughborough University, Loughborough, UK, 2003. 